\title{
A Facile Method for Simultaneously Measuring Neuronal Cell Viability and Neurite Outgrowth
}

\author{
Michael K. Hancock ${ }^{1, *}$, Leisha Kopp ${ }^{1}$, Navjot Kaur ${ }^{2}$ and Bonnie J. Hanson ${ }^{1}$ \\ Life Sciences Solutions Group, Thermo Fisher Scientific, ${ }^{1}$ Madison, WI 53719, ${ }^{2}$ Frederick, MD 21704
}

\begin{abstract}
Neurite outgrowth is an important morphological phenotype of neuronal cells that correlates with their function and cell health, yet there are limited methods available for measuring this phenomenon. Current approaches to measuring neurite outgrowth are laborious and time-consuming, relying largely upon immunocytochemical staining of neuronal markers (e.g., beta-III tubulin or MAP2) followed by manual or automated microscopy for image acquisition and analysis. Here we report the development of a quick and simple dual-color fluorescent dye-based staining method that allows for the simultaneous measurement of neuronal cell health and relative neurite outgrowth from the same sample. An orangered fluorescent dye that stains cell membrane surfaces is used as an indirect reporter of changes in relative neurite outgrowth due to alterations in the number or length of membrane projections emanating from neuronal cell bodies. Cell viability is assessed simultaneously via the use of a cell-permeant dye that is converted by intracellular esterase activity from a non-fluorescent substrate to a green-fluorescent product. Using Neuroscreen-1 cells (a PC-12 subclone), primary rat cortex neurons, and human induced pluripotent stem cell (iPSC)-derived neurons, we demonstrate that this multiplex assay allows for rapid visualization and unbiased, quantitative plate reader analysis of neuronal cell health and neurite outgrowth.
\end{abstract}

Keywords: Cell membrane stain, fluorescence, multiplex assay, neural cell viability, neurite outgrowth, plate reader.

\section{INTRODUCTION}

Measuring changes in neurite outgrowth is one of the most widely performed phenotypic assays since the ability of neuronal cells to project membrane extensions from their cell bodies is closely linked to their function and cell health [1, 2]. Of particular recent interest has been the realization that alterations in neuronal cell function underlie a number of cognitive and neurodegenerative disorders (e.g., Parkinson's and Alzheimer's), leading to intensive efforts to identify molecules that may be able to prevent or ameliorate these conditions [3, 4]. Additionally, there is a need for improved neuronal cell-based models and methods to monitor the neurotoxicity effects of chemical and environmental agents in routine testing and high-throughput drug screening campaigns $[5,6]$. While a number of cytotoxicity assays exist, techniques with simplified workflows that enable rapid approximations of changes in neurite outgrowth are lacking in the field.

Current approaches to measuring neurite outgrowth rely largely upon immunocytochemistry (ICC) for neuronal markers (e.g., beta-III tubulin or MAP2) followed by manual or automated image acquisition and analysis, altogether which requires several hours and numerous manipulations to go from sample to results (Fig. 1A) [7, 8]. For instance, a

*Address correspondence to this author at the Cellular Dynamics International, 525 Science Drive, Madison, WI 53711, USA; Tel: 608-692-0311;

E-mail: mhancock@cellulardynamics.com typical ICC workflow involves many liquid-handling steps, e.g., cell fixation, permeabilization and blocking, primary and secondary antibody incubations, with multiple washes between each step. Microscopic visualization or high-content imaging is subsequently performed to acquire representative fields of view. Selected images are then processed either manually or via software tools that allow for the digital reconstruction and tracing of neurons and their extensions [9, 10]. Although significant in-roads have been made in recent years to improve the robustness of neuronal image acquisition and analysis, these methods remain computationally challenging and being image-based are inherently subjective in nature [7].

To overcome many of the limitations of current ICC/image-based techniques for staining neuronal cultures and measuring changes in neurite outgrowth, we report the development of a quick and simple fluorescent dye-based staining method that is suitable not only for microscopic visualization, but also enables unbiased microplate reader quantification. Moreover, this dual-color stain allows for the simultaneous assessment of neuronal cell health and relative neurite outgrowth from the same sample (Figs. 2 and 3). A typical workflow using this kit requires less than 30 minutes and two media exchange steps prior to taking a measurement (Fig. 1B). We demonstrate application of this neuronal cell health/neurite outgrowth multiplex assay by measuring the effects of growth stimulating culture conditions and known neurotoxic agents upon primary rat and human induced pluripotent stem cell (iPSC)-derived neurons in addition to PC12 subclone Neuroscreen-1 cells. 


\section{A}

\begin{tabular}{l}
$\mid=20$ \\
\hline Fix \\
Traditional immunocytochemistry
\end{tabular}

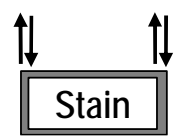

Neurite outgrowth + viability stain

B

\section{Optional: pre-fix cells to improve adherence} (add $4 \%$ formaldehyde)

\section{Step 1: Remove cell medium \& add stain solution}

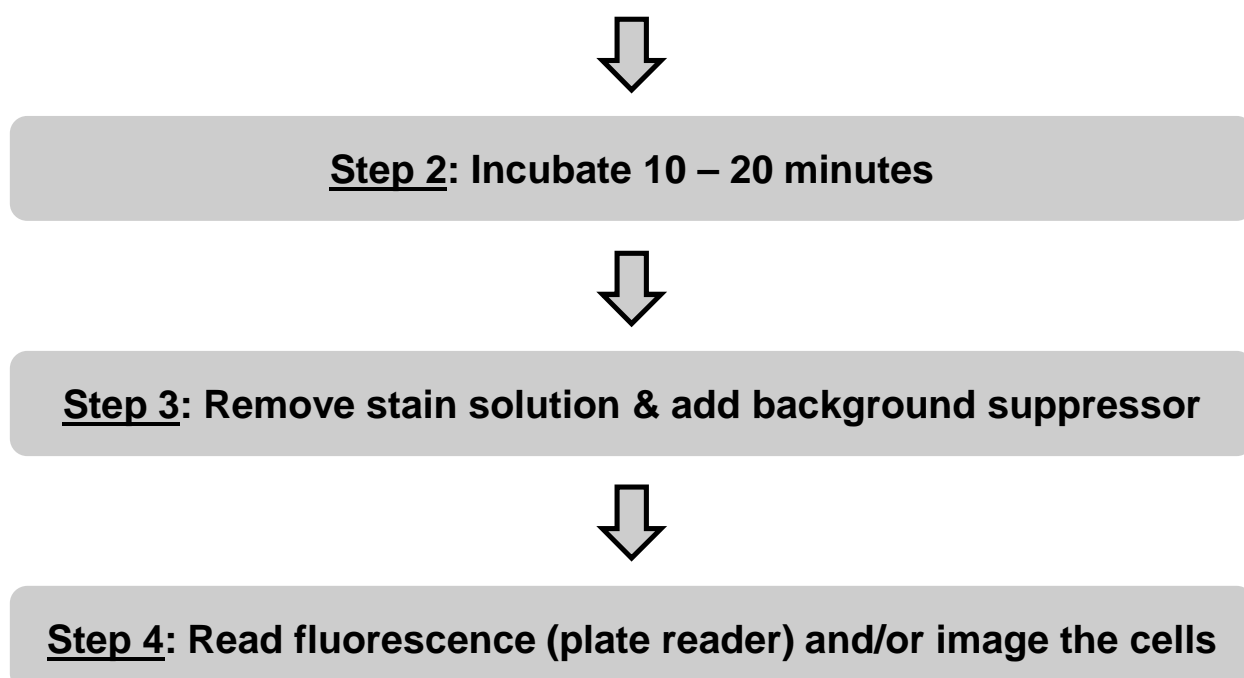

Fig. (1). Workflow comparison of the proposed dye-based neurite outgrowth assay with traditional immunocytochemistry. (A) Traditional immunocytochemistry (antibody-based staining) for neurite outgrowth requires numerous pipette steps (arrows) and several hours to process. In contrast, the dye-based staining method in this report uses a streamlined workflow that typically requires approximately 30 minutes to complete. (B) Outline of the neurite outgrowth assay workflow. 
A
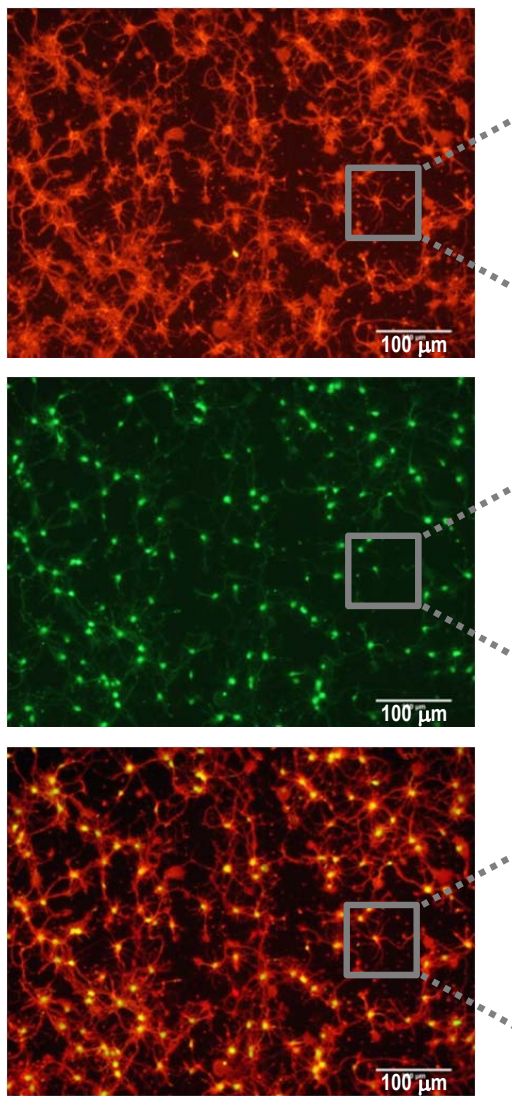

B
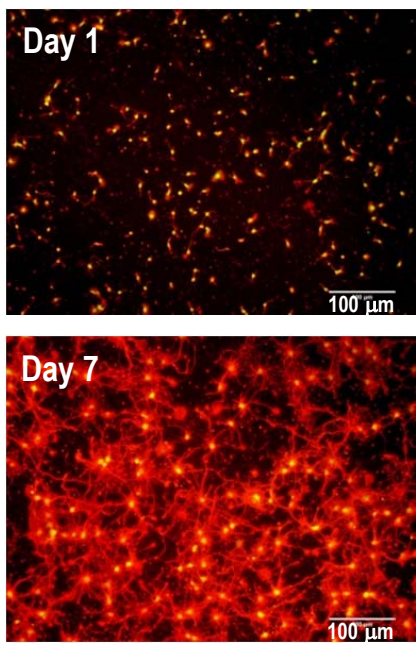

Cell Viability Indicator

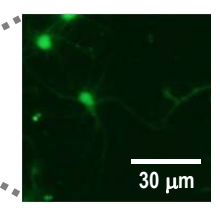

C

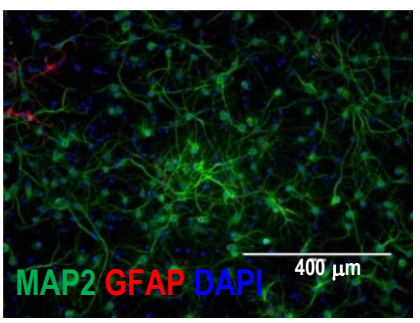

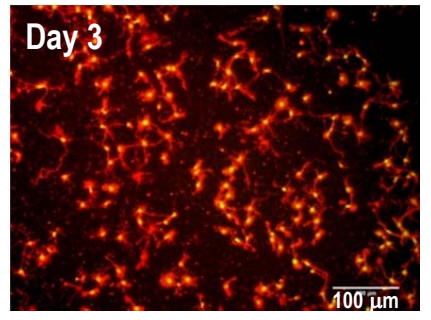
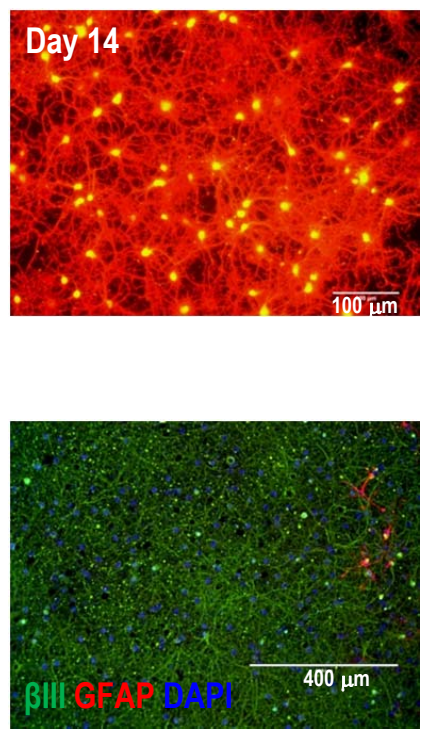

Fig. (2). Multiplex fluorescent dye-based staining of neurite outgrowth and neuronal cell health. (A) A 7-day culture of rat cortex neurons was stained using an orange-red fluorescent Cell Membrane Stain, which serves as a reporter of neurite outgrowth, along with a greenfluorescent Cell Viability Indicator that requires intracellular esterase activity. (B) Cryopreserved rat cortex neurons were plated and cultured under neurite outgrowth-promoting conditions, then co-stained with the Cell Membrane Stain (orange) and the Cell Viability Indicator (green) at different time-points. (C) Immunocytochemistry analysis of the rat cortex neuron cultures for neuronal markers MAP2 and beta-III tubulin and glial marker GFAP.

\section{MATERIALS AND METHODS}

\section{Neuronal Cell Culture}

Cryopreserved rat cortex neurons (Life Technologies, Carlsbad, CA) were thawed and plated onto clear-bottom poly-D-Lysine 96-well plates (BD Biosciences, San Jose, CA) at a cell plating density of $\sim 5,000$ to $\sim 20,000$ cells/well depending on the post-thaw cell viability. Rat cortex neurons were grown in Neurobasal medium (Life Technologies) supplemented with B-27 serum free supplement (Life Technologies) and $0.5 \mathrm{mM}$ GlutaMAX (Life Technologies). PC12 derivative Neuroscreen-1 cells were obtained from Thermo Fisher Scientific (Waltham, MA) and plated onto collagen I-treated 96-well plates (Life Technologies) at $\sim 10,000$ cells/well. Neuroscreen-1 cells were cultured in RPMI 1640 (Life Technologies) supplemented with 10\% fetal bovine serum (Life Technologies) and $100 \mathrm{U} / \mathrm{mL}$ Penicillin $/ 100 \mu \mathrm{g} / \mathrm{mL}$ Streptomycin (Life Technologies). To stimulate neurite outgrowth, mouse nerve growth factor $2.5 \mathrm{~S}$ (NGF 2.5S, Life Technologies, Carlsbad, CA) was added at the indicated concentrations to the Neuroscreen- 1 cells at the same time they were plated. Cryopreserved human iPSCderived iCell Neurons (Cellular Dynamics International,
Madison, WI) were thawed, plated ( 10,000 cells/well), and maintained in accordance with the manufacturer's protocol on black-wall, clear-bottom 96-well plates (Corning, Corning, NY) that were coated with a base layer of poly-Lornithine (Sigma, Saint Louis, MO) and a top layer of laminin (Sigma).

\section{Compound Treatment}

Compounds were obtained as follows: Nocodazole (Thermo Fisher Scientific), Staurosporine (Life Technologies), SAHA (US Biological, Salem, MA), Antimycin A and CCCP (Sigma). Compounds were prepared at $1000 \mathrm{X}$ in DMSO (Sigma) and then diluted into the respective cell culture medium such that the final amount added to the cells did not exceed $0.1 \%$.

\section{Multiplex Neurite Outgrowth/Cell Viability Assay}

Dual-color staining for relative neurite outgrowth (via cell membrane labeling) or neuronal cell health (via cell viability/esterase activity assessment) was performed using components of the Neurite Outgrowth Staining Kit (Life Technologies), which contains three dyes: a Cell Membrane 

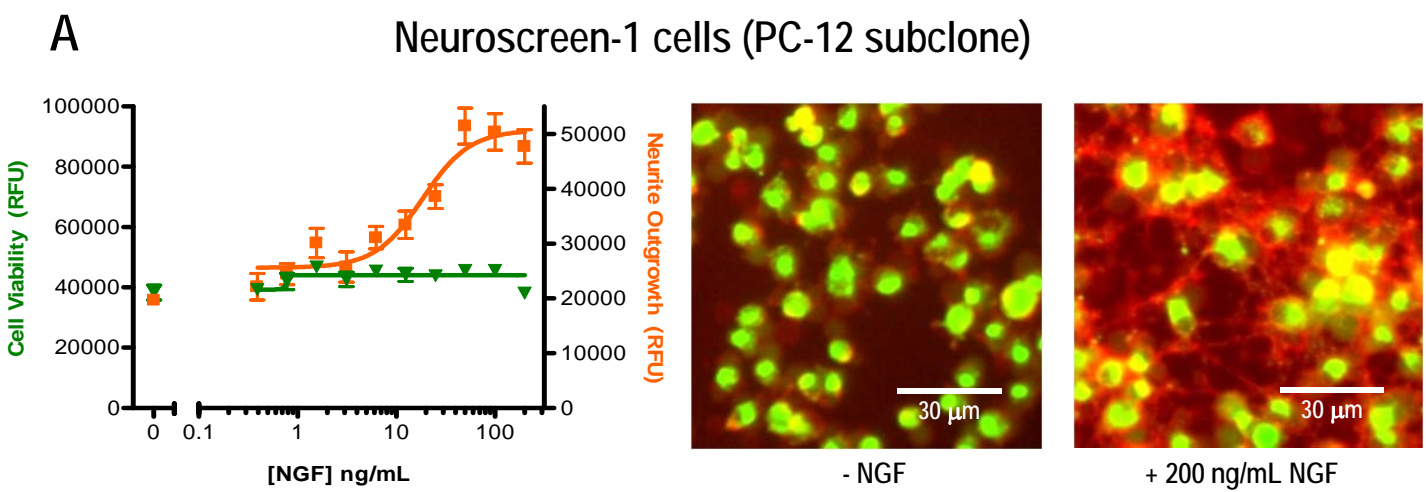

B
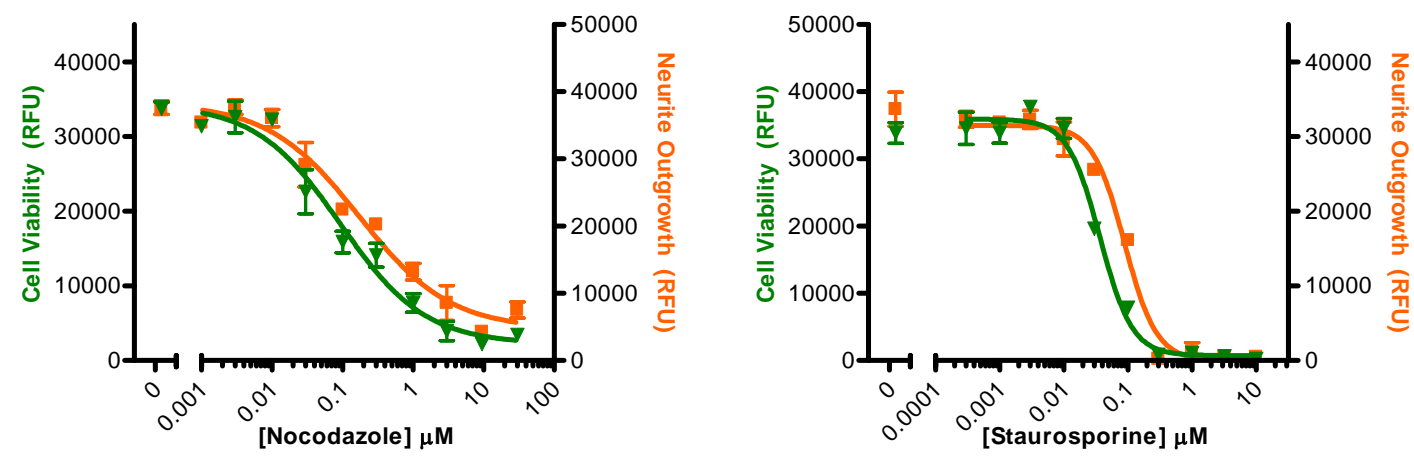

Fig. (3). Plate reader quantification of relative neurite outgrowth and cell viability using Neuroscreen-1 (PC-12 subclone) cells. (A) Cells were plated in 96-well format and treated with a serial dilution of nerve growth factor (NGF) for 4 days. Prior to staining them, the cells were fixed with $4 \%$ formaldehyde to improve their adherence to the assay plate. Relative cell viability $(\boldsymbol{\nabla})$ and neurite outgrowth ( $(\mathbf{})$ were measured using a bottom-read fluorescence microplate reader to detect the green and orange-red fluorescence intensity from each well. Data plotted are the mean \pm SEM ( $n=4$ replicate wells) of one representative experiment of three independent experiments that were performed. For visual reference, representative merge images are also shown. (B) In a separate experiment, cells were plated in the presence of $100 \mathrm{ng} / \mathrm{mL}$ NGF and then treated with titrations of known cytotoxic compounds Nocodazole and Staurosporine for 3 days (data are mean \pm SEM, triplicate wells).

Stain (TRITC filter-compatible), calcein AM (Cell Viability Indicator, FITC filter-compatible), and a Background Suppressor reagent. A typical staining procedure is briefly outlined in Fig. 1B, with additional details as follows. A 1x staining solution was prepared by diluting the Cell Membrane Stain and Cell Viability Indicator dyes (provided at 1000x) together in either PBS or Live Cell Imaging Solution (Life Technologies). Next, the growth medium was removed from the wells and replaced by adding $1 \mathrm{x}$ staining solution (100 $\mu \mathrm{L} / w e l l$, 96-well format) to the wells. Assay plates were incubated at either room temperature or $37^{\circ} \mathrm{C}$, typically for $10-20$ minutes or until sufficient green fluorescence from the converted Cell Viability Indicator was detected. Following the incubation step, the stain solution was removed and replaced with 1x Background Suppressor (provided at 100x), also diluted in PBS or Live Cell Imaging Solution. The assay plates were subjected to fluorescence plate reader quantification and microscopic imaging as described below. Whereas rat cortex neurons adhered tightly to their culture plates, we observed that Neuroscreen-1 and iCell Neurons were less securely adhered to their respective culture plates. Accordingly, to minimize potential cell loss during the two media exchange staining steps, these cells were pre-fixed prior to staining by adding an equal volume of $8 \%$ formaldehyde (diluted in PBS) onto the assay wells containing cells and growth medium and incubating for $\sim 5-$ 15 minutes at room temperature. The staining solution was also supplemented with $4 \%$ formaldehyde. Note that although these cells were technically no longer viable following fixation, sufficient esterase activity was maintained to efficiently convert the Cell Viability Indicator to green fluorescent product and thereby still provide a useful cell health indication of the relative number of "healthy" cells without compromised cell membranes present in the assay wells just prior to fixation and staining. For example, despite fixative being added to the cells before applying the Cell Viability Indicator the vehicle control cells retained enough esterase activity to generate a strong fluorescent signal, indicative of the presence of a relatively large population of viable cells without compromised membranes at the time the cells were fixed, in contrast to the dose-dependent loss of signal obtained from the test samples that had been treated with cytotoxic compounds Nocodazole or Staurosporine (Fig. 3B). Since the iCell Neurons, when fixed, tended to more slowly convert the Cell Viability Indicator they were either incubated longer or a $2 \mathrm{x}$ amount of the Cell Viability Indicator was applied to these cells. Fixed cells could also be stored for later analysis. 


\section{Fluorescence Plate Reader Detection and Analysis}

Relative cell viability and neurite outgrowth was measured using a Tecan Safire ${ }^{2}$ fluorescence plate reader (Tecan Group Ltd., Maennedorf, Switzerland) to detect the fluorescence intensity from each well, expressed in relative fluorescence units (RFU). Bottom-read fluorescence detection was performed using monochromator excitation/emission settings of 483/525 nm (12 nm bandwidths) for the green fluorescent Cell Viability Indicator and 554/567 $\mathrm{nm}(5 \mathrm{~nm}$ bandwidths) for the orange-red fluorescent Cell Membrane Stain. Cellfree controls were included and used for background subtraction. Unless stated otherwise, cell treatments were typically performed in triplicate, from which the mean \pm SEM was plotted. Curve fitting was performed with GraphPad Prism (GraphPad Software, La Jolla, CA) using a nonlinear regression equation for variable slope sigmoidal dose-response to estimate $50 \%$ effective or inhibitory concentration $\left(\mathrm{EC}_{50}\right.$ or $\left.\mathrm{IC}_{50}\right)$ values.

\section{Imaging}

The antibodies used for immunocytochemistry were obtained from Life Technologies. Cells were visualized using a Zeiss Axiovert $25 \mathrm{CFL}$ inverted fluorescence microscope (Carl Zeiss, Oberkochen, Germany) and standard FITC or TRITC filter sets. Green and orange-red fluorescent images were captured using an integrated Pixera Penguin 600CL camera (Pixera, San Jose, CA) and processed using ImageJ software (NIH, http://imagej.nih.gov/ij) to create merged picture overlays.

\section{RESULTS AND DISCUSSION}

Neurite outgrowth is commonly monitored via immunocytochemistry followed by microscopic image acquisition and analysis, procedures which are both time-consuming and tedious (Fig. 1A) [7, 8]. To generate a simpler method (Fig. 1B) for rapidly approximating neurite outgrowth in addition to neuronal cell health, a select combination of fluorescent dyes and a background suppression reagent was evaluated as follows. First, since changes in neurite outgrowth affect the number and/or length of the membrane projections emanating from neuronal cell bodies (i.e., alters the total amount of cell membrane surface area), a non-penetrating, bright orange-red fluorescent dye (Cell Membrane Stain; excitation/emission $\sim 555 \mathrm{~nm} / \sim 565 \mathrm{~nm}$ ) that evenly coats the outer surface of cells was selected as a non-specific neurite outgrowth reporter. Both the cell body and any neurite projections are uniformly stained with this dye (Fig. 2A). Because this relatively sticky dye can also coat plastic tissue-culture surfaces, we found that adding a background suppression reagent following removal of the stain minimizes this background fluorescence and thereby eliminates the need for additional wash steps (Fig. 1B).

The suitability of this dye-based approach to stain for neurite outgrowth was tested by setting up a time-course in which cryopreserved rat cortex neurons were plated under neurite outgrowth-promoting culture conditions and then stained at several different time-points up to 14 days after initiating the culture (Fig. 2B). As expected, increased cell membrane staining was observed over time, indicative of significant neurite outgrowth occurring within this culture. It is important, however, to point out that since the Cell Membrane Stain is not neuron-specific it will label any cell-type, which precludes its use for discriminating between neuronal and non-neuronal cell types in mixed culture settings. Nevertheless, this cell membrane staining approach is suitable for mono-cultures in which a single cell-type (e.g., PC-12 cells) or a highly-enriched neuronal cell population (e.g., Fig. 2C, which indicates that most of the cells in the rat cortex neuron cultures used in these experiments stained positive for the neuronal markers MAP2 and beta-III tubulin, but not for the glial marker GFAP) is interrogated for phenotypic changes relating to cell membrane content or morphological presentation such as, but certainly not limited to, neurite outgrowth.

Since neurite outgrowth is closely linked to neuronal cell health, it would be ideal to measure both parameters from the same sample. Accordingly, the acetomethoxy derivate of calcein (Calcein AM), a well-established cell viability reagent [11], was selected for inclusion alongside the Cell Membrane Stain. This cell-permeable dye is converted by intracellular esterase activity from a non-fluorescent substrate to a green-fluorescent product (Cell Viability Indicator; excitation/emission $\sim 495 \mathrm{~nm} / \sim 515 \mathrm{~nm}$, Fig. 2A). Notably, application of the Cell Viability Indicator reagent to neuronal cells results in differential staining, with relatively bright staining of the cell body and dim labeling of the neurite extensions. This proves advantageous in that the majority of the green fluorescent signal produced corresponds with the core cell body and thereby allows for easy visual discrimination of the individual viable cells from amongst the neurite outgrowth tangles. While technically no longer viable, we also found that healthy cells (i.e., those with intact cell membranes) that were pre-fixed with formaldehyde to improve their adherence prior to staining still retained sufficient esterase activity to convert the Cell Viability Indicator to green fluorescent product (e.g., Fig. 3A), thereby providing a "snapshot" of the health of the culture at the time of cell fixation. The combination of the Cell Viability Indicator dye with the Cell Membrane Stain enabled simultaneous monitoring of cell health and relative neurite outgrowth within the same sample (e.g., Fig. 2A, merged image).

An analogous fluorescent dye-based staining approach to measuring neurite outgrowth (using HCS CellMask Red, which stains the entire cell and not just the outer cell membrane surface) was recently reported [8]. This study demonstrated that dye-based labeling was clearly a faster and easier staining alternative to either anti-beta-III tubulin antibody labeling or green fluorescent protein (GFP) expression when performing high-content imaging. However, since this work relied upon visualization the benefits from applying a simplified staining workflow were largely offset by the extensive downstream image acquisition and analysis requirements. For instance, high content imaging typically involves the use of various algorithms, artifact filtering and signal intensity thresholds to identify and differentiate the target cell segmentations to be analyzed from representative images. In contrast, fluorescence plate readers simply detect the 
Table 1. Compound $\mathrm{IC}_{50}(\mu \mathrm{M})$ values by neuronal cell type and test mode.

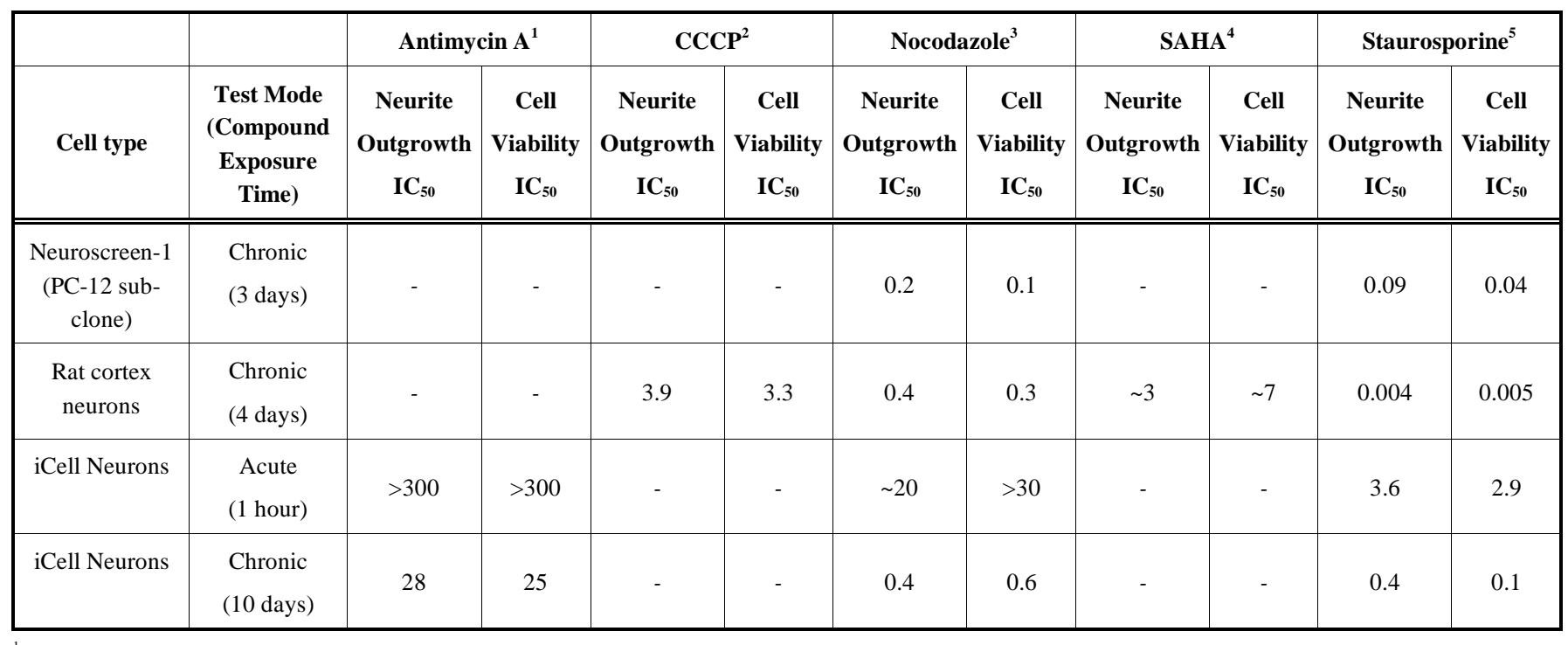

${ }^{1}$ Antimycin $\mathrm{A}$ is a mitochondrial oxidative phosphorylation inhibitor

${ }^{2} \mathrm{CCCP}$ (carbonyl cyanide $m$-chlorophenyl hydrazone) is a mitochondrial oxidative phosphorylation inhibitor

${ }^{3}$ Nocodazole is a microtubule polymerization inhibitor, mitotic inhibitor

${ }^{4}$ SAHA (suberoylanilide hydroxamic acid) is a histone deacetylase (HDAC) inhibitor

${ }^{5}$ Staurosporine is a pan-kinase inhibitor

fluorescence intensity emitted from the assay well. Here we focused on extending the utility of quick and simple dyebased stains beyond expediting sample preparation for imaging to also enabling rapid, unbiased fluorescent plate readerbased approximations of neurite outgrowth.

Although the suitability of monitoring neuronal cell health via plate reader determinations (besides imaging) has been previously demonstrated using calcein AM [12], to our knowledge this has not been shown using cell membrane staining reagents. Our premise here was that neurite outgrowth-mediated fluctuations in Cell Membrane Stainaccessible surface area would correspond with detectable changes in the orange-red signal intensity. Likewise, changes in neuronal cell health affecting esterase levels or cell membrane integrity would result in changes to the Cell Viability Indicator signal. To test this concept we stained a monoculture of PC-12 derivative Neuroscreen-1 cells. The PC-12 cell-line (originating from a rat pheochromocytoma) is commonly used as a neuronal differentiation model since it responds to nerve growth factor (NGF) to generate neuritelike extensions $[5,6]$. When cells were treated with a serial titration of NGF, cell membrane staining resulted in a dosedependent increase in orange-red fluorescence that was detected by the plate reader and could also be visually confirmed (Fig. 3A). Moreover, the estimated $\mathrm{EC}_{50}$ of $\sim 18$ $\mathrm{ng} / \mathrm{mL}$ for NGF was within the anticipated working range based on the manufacturer's specification (i.e., the NGF certificate of analysis states: "Half-maximal neurite outgrowth must occur in $\mathrm{PC}-12$ cells at $\leq 50 \mathrm{ng} / \mathrm{ml}$ NGF $2.5 \mathrm{~S}$ "). Whereas an increase in relative neurite outgrowth was detected, minimal fluctuations in the green fluorescent viability signal were observed, indicating that cell health was maintained across the NGF dose range (Fig. 3A). When cytotoxic compounds Nocodazole and Staurosporine were applied to the cells, a dose-dependent loss-of-signal was observed in both the orange-red and green fluorescence emission channels (Fig. 3B). The calculated $\mathrm{IC}_{50}$ values (Table 1) for both compounds (and both reporter dyes) were in line with their known sub-micromolar bioactive concentrations. In addition, Z' factor values, commonly used to assess assay quality by accounting for both the assay window as well as the assay variation [13], were calculated for the $30 \mu \mathrm{M}$ Nocodazole (Z' factor $=0.60$ for the Cell Membrane Stain, 0.76 for the Cell Viability Indicator) and the $10 \mu \mathrm{M}$ Staurosporine ( $Z^{\prime}$ factor $=0.59$ for the Cell Membrane Stain, 0.76 for the Cell Viability Indicator) treatments relative to the untreated control. In these two examples the Z' factor values for both reporter dyes exceeded the 0.5 minimum threshold typically reported for cell-based HTS assays, suggesting that this dual reporter assay could be useful for screening applications. Together these test data strongly indicate the potential for using both fluorescent reporter dyes, i.e., cell membrane staining as a neurite outgrowth reporter and esterasedependent conversion of calcein AM as a cell health indicator, to generate a multiplex assay suitable for rapid, unbiased plate reader quantification.

To further examine this rapid stain-and-read approach to measuring changes in neurite outgrowth and cell viability, we tested the effects of a small set of compounds with widely varying mechanisms of action (Table 1) in two additional neuronal cell models: primary rat cortex neurons and human iPSC-derived neurons. Cryopreserved rat cortex neurons were thawed, plated, and allowed to recover and undergo neurite outgrowth for a week prior to applying the compounds for an additional 4 days. The resultant 11-day culture exhibited substantial neurite outgrowth (Fig. 4A) that was reduced to varying degrees by the mechanistically distinct compounds (Fig. 4). As seen with the Neuroscreen-1 


\section{A}

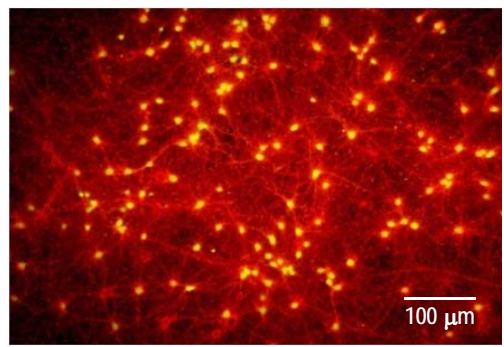

Untreated control

B

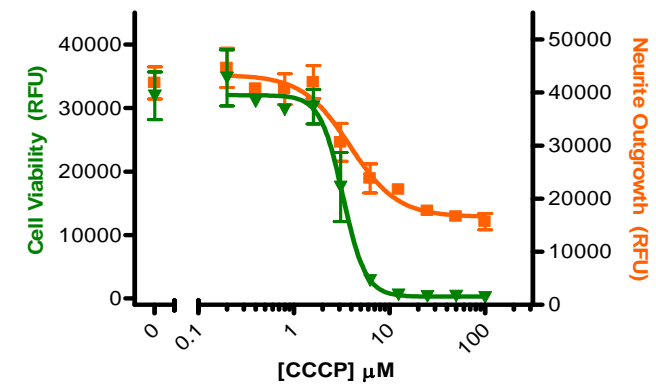

C

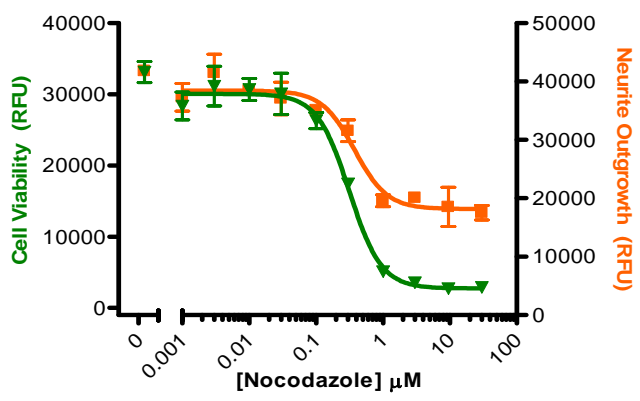

D

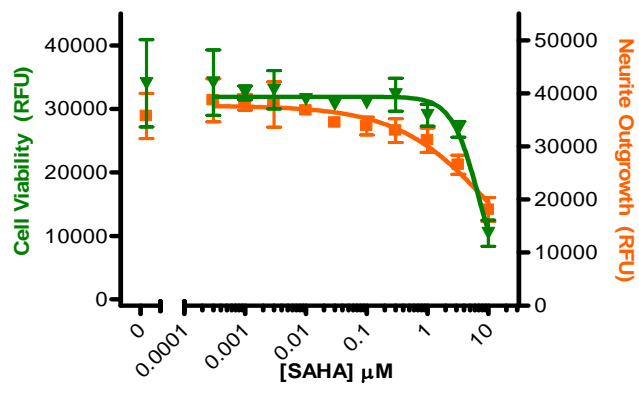

E

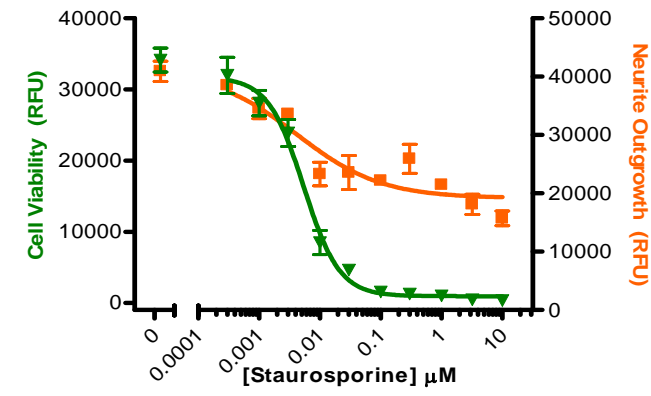

\section{Rat cortex neurons}

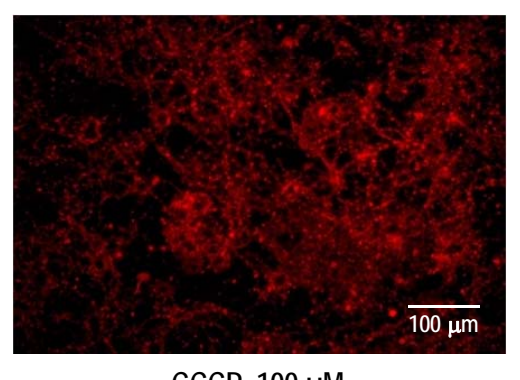

CCCP, $100 \mu \mathrm{M}$

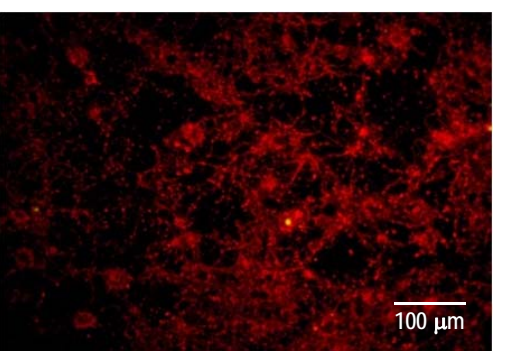

Nocodazole, $30 \mu \mathrm{M}$

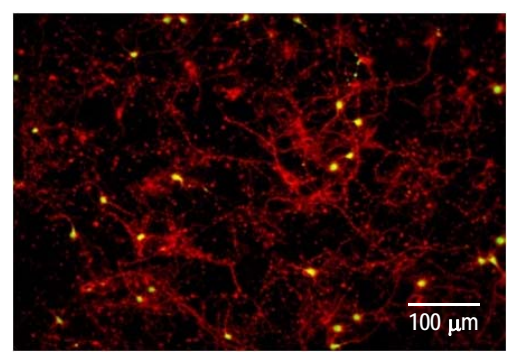

SAHA, $10 \mu \mathrm{M}$

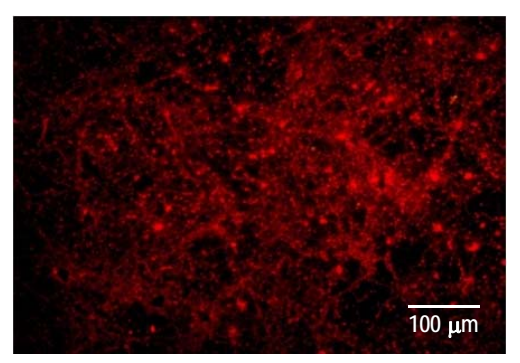

Staurosporine, $10 \mu \mathrm{M}$

Fig. (4). Plate reader quantification of relative neurite outgrowth and cell viability using primary rat cortex neurons. Cryopreserved cells were thawed and plated in 96-well format, cultured for 7 days, and then treated with compounds for an additional 4 days prior to assaying them. Relative cell viability $(\boldsymbol{\nabla})$ and neurite outgrowth (-) were measured using a bottom-read fluorescence microplate reader (data plotted are the mean \pm SEM of triplicate wells) and representative images were taken of the following conditions: (A) untreated control, or maximum test concentration for (B) CCCP, (C) Nocodazole, (D) SAHA, and (E) Stauorosporine. 

3
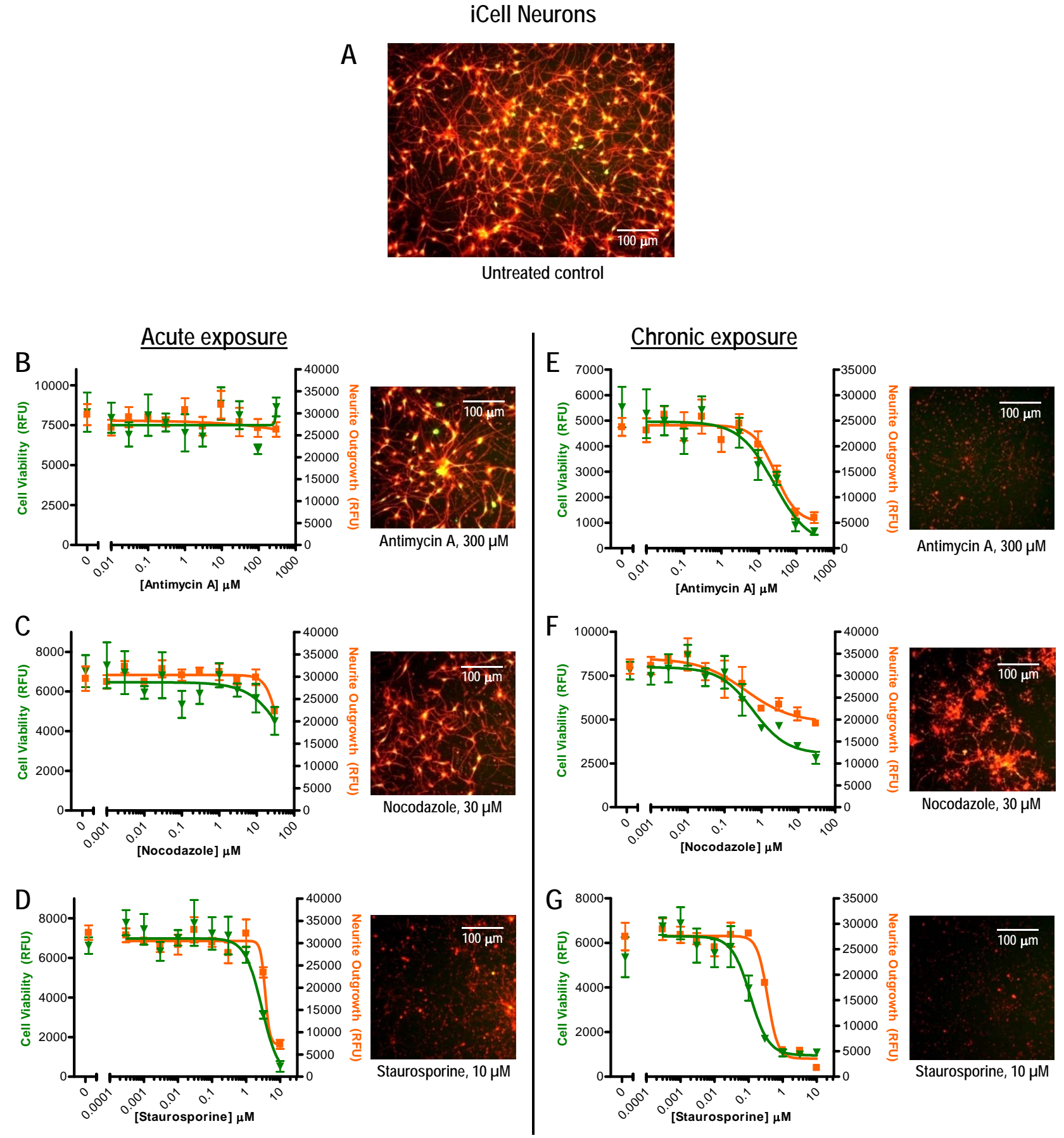

Fig. (5). Plate reader quantification of relative neurite outgrowth and cell viability using human iPSC-derived iCell Neurons. Cryopreserved cells were thawed, plated in 96-well format and allowed to recover overnight. The next day, test compounds were either applied once for 1 hour (acute exposure) and then replaced with fresh medium thereafter, or applied and left on (chronic exposure) and replenished during subsequent feedings (every $3-4$ days). After 11 days in culture, the cells were pre-fixed with $4 \%$ formaldehyde, stained, and relative cell viability $(\boldsymbol{\nabla})$ and neurite outgrowth ( $\mathbf{a})$ were measured using a bottom-read fluorescence microplate reader (data plotted are the mean \pm SEM of triplicate wells). Data plots and selected compound-treated images are as follows: (A) untreated control, (B and E) Antimycin A, (C and F) Nocodazole, (D and G) Stauorosporine.

cells, Nocodazole and Staurosporine were potent, submicromolar inhibitors of neurite outgrowth with concomitant effects on cell health (Figs. 4C and 4E, Table 1). Interestingly, whereas the $\mathrm{IC}_{50}$ values for Nocodazole (a microtubule inhibitor) were similar to those obtained with Neuroscreen-1 cells, the $\mathrm{IC}_{50}$ values of Staurosporine (a general kinase 
Table 2. Compound dose-response curve Hill slope values.

\begin{tabular}{|c|c|c|c|c|c|c|c|c|c|c|c|}
\hline \multirow[b]{2}{*}{ Cell Type } & \multirow[b]{2}{*}{$\begin{array}{c}\text { Test Mode } \\
\text { (Compound } \\
\text { Exposure Time) }\end{array}$} & \multicolumn{2}{|c|}{ Antimycin A } & \multicolumn{2}{|c|}{ СССР } & \multicolumn{2}{|c|}{ Nocodazole } & \multicolumn{2}{|c|}{ SAHA } & \multicolumn{2}{|c|}{ Staurosporine } \\
\hline & & $\begin{array}{c}\text { Neurite } \\
\text { Outgrowth }\end{array}$ & $\begin{array}{c}\text { Cell } \\
\text { Viability }\end{array}$ & $\begin{array}{c}\text { Neurite } \\
\text { Outgrowth }\end{array}$ & $\begin{array}{c}\text { Cell } \\
\text { Viability }\end{array}$ & $\begin{array}{c}\text { Neurite } \\
\text { Outgrowth }\end{array}$ & $\begin{array}{c}\text { Cell } \\
\text { Viability }\end{array}$ & $\begin{array}{c}\text { Neurite } \\
\text { Outgrowth }\end{array}$ & $\begin{array}{c}\text { Cell } \\
\text { Viability }\end{array}$ & $\begin{array}{c}\text { Neurite } \\
\text { Outgrowth }\end{array}$ & $\begin{array}{c}\text { Cell } \\
\text { Viability }\end{array}$ \\
\hline $\begin{array}{c}\text { Neuro- } \\
\text { screen-1 } \\
\text { (PC-12 } \\
\text { subclone) }\end{array}$ & $\begin{array}{l}\text { Chronic } \\
\text { (3 days) }\end{array}$ & - & - & - & - & -0.7 & -0.7 & - & - & -1.7 & -1.7 \\
\hline $\begin{array}{l}\text { Rat cortex } \\
\text { neurons }\end{array}$ & $\begin{array}{l}\text { Chronic } \\
\text { (4 days) }\end{array}$ & - & - & -1.7 & -3.7 & -1.8 & -1.8 & -0.6 & -1.9 & -0.6 & -1.5 \\
\hline $\begin{array}{c}\text { iCell } \\
\text { Neurons }\end{array}$ & $\begin{array}{l}\text { Acute } \\
\text { (1 hour) }\end{array}$ & n.d. & n.d. & - & - & n.d. & n.d. & - & - & -6.5 & -1.8 \\
\hline $\begin{array}{c}\text { iCell } \\
\text { Neurons }\end{array}$ & $\begin{array}{l}\text { Chronic } \\
\text { (10 days) }\end{array}$ & -1.5 & -0.9 & - & - & -0.7 & -1.0 & - & - & -2.8 & -1.6 \\
\hline
\end{tabular}

inhibitor) were nearly an order of magnitude lower, suggesting that rat cortex neurons may be more sensitive to kinase inhibition than PC-12 derivative Neuroscreen-1 cells. The additional two compounds tested in rat cortex neurons (but not Neuroscreen-1 cells), mitochondrial oxidative phosphorylation inhibitor CCCP and HDAC inhibitor SAHA, yielded low $\mu \mathrm{M} \mathrm{IC} 50$ values (Figs. $4 B$ and $4 \mathrm{D}$, Table 1) that were in accordance with their known bioactivities [14]. Also notable was that for each of the four compounds the estimated $\mathrm{IC}_{50}$ values for both the Cell Membrane Stain and the Cell Viability Indicator readouts appeared fairly similar (i.e., within $\sim 2$-fold, Table 1), indicating that loss of neurite outgrowth was closely linked with decreased cell health.

By juxtaposing the neurite outgrowth and cell health readouts together within the same graph, a few additional observations could be made from the rat cortex neurotoxicity example data set. First, for the Cell Viability Indicator readout, treatment concentrations leading to complete loss of cell health also resulted in complete loss of plate reader detectable green fluorescence signal above background (i.e. RFU values approaching 0). In contrast, there appeared to be no greater than a $\sim 2$-fold maximal loss of the neurite outgrowth reporter signal relative to the untreated control (i.e., drop from $\sim 30,000$ to $\sim 15,000$ RFUs), implying that a significant amount of cell membrane-stained material persisted even under very cytotoxic conditions. Indeed, visual inspection confirmed the plate reader results, with varying patterns of what appeared to be stained neurite network disintegration and cell debris remaining in the compound treated wells (e.g., compare the images from Figs. $4 \mathbf{B}-\mathbf{E}$ with the untreated control in Fig. 4A). These results suggested that in order to increase the cell membrane staining assay sensitivity it would be better to apply compounds earlier (i.e., soon after plating the cells) to identify neurotoxic conditions that prevent neurite outgrowth instead of applying them after extensive networks had already been established (this hypothesis was tested in the experiment described below in Fig. 5).
Additionally, by overlaying the compound dose-response curves from both readouts it became apparent that in certain cases, e.g., Nocodazole treatment (Fig. 4C), the Hill slopes (Table 2) were nearly identical whereas in other cases, e.g., SAHA and Staurosporine, there were some noticeable differences that could have cause-and-effect implications. For instance, we speculate that for SAHA-treated rat cortex neuronal cells the gradual dose-dependent loss of neurite outgrowth may have preceded the abrupt (albeit incomplete) drop-off in cell health observed at the highest concentration tested (Fig. 4D). Conversely, for Staurosporine-treated cells we speculate that loss of cell health may have occurred prior to and/or more quickly than the retraction or dissolution of the neurite extensions (Fig. 4E). While additional work beyond the scope of this report will be required to confirm these suggestions, taken together these data examples illustrate the potential utility of the stain-and-read approach to neurite outgrowth and cell health in order to quickly flag interesting conditions for detailed follow-up.

Recent advances in the development of human iPSCderived neuron cell models offer the potential for generating and comparing normal and disease patient neurons to uncover mechanisms of action and identify potential therapeutic strategies. We tested one commercially available model, the iCell Neuron [4, 15], with our neurite outgrowth/cell health multiplex assay approach. We designed this experiment to compare two scenarios: acute (1 hour) compound treatment near the beginning of the culture versus sustained chronic exposure (10 days) for the duration of the culture (Fig. 5). For the cultures treated briefly with compound and then allowed to recover, only Staurosporine treatment exhibited strong deleterious effects on neurite outgrowth and cell health (Figs. 5B - D). Chronic exposure, however, resulted in detection of substantial neurotoxic effects for all three test compounds (Figs. 5E - G), with a marked left-shift in the Staurosporine dose-response curve relative to the acute 
exposure (Table 1). Again, the plate reader results were consistent with the images taken, including the noticeably higher bottom plateau that was obtained with chronic Nocodazole treatment (Fig. 5F) versus the nearly complete loss of both reporter signals for Staurosporine and mitochondrial oxidative phosphorylation inhibitor Antimycin A (Figs. 5E and 5G). Notably, these iPSC-derived neurons exhibited similar chronic exposure sensitivity (i.e., similar neurite outgrowth $\mathrm{IC}_{50}$ values, Table 1) to Nocodazole as the other two cell types. However, like PC-12 derivative Neuroscreen-1 cells, iCell Neurons appeared to be much less sensitive to lower Staurosporine doses (e.g., $0.01 \mu \mathrm{M}$ ) than rat cortex neurons. These data support the notion that in addition to exposure time, compound sensitivities may depend upon the unique characteristics of the cell cultures being interrogated. Interestingly, the neurite outgrowth plate reader results (Table 1) obtained here for iCell Neurons with Antimycin A $\left(\mathrm{IC}_{50} \sim 28 \mu \mathrm{M}\right)$ and Staurosporine $\left(\mathrm{IC}_{50} \sim 0.4\right.$ $\mu \mathrm{M})$ compare well to those previously reported for these same cells using a traditional high content image-based analysis approach (Antimycin $\mathrm{A} \mathrm{IC}_{50} \sim 13 \mu \mathrm{M}$, Staurosporine $\mathrm{IC}_{50} \sim 0.9 \mu \mathrm{M}$ ) [16]. Taken together, these results confirm the potential for the dye-based plate reader approach described in this report to enable rapid approximations of neurite outgrowth and cell viability.

\section{CONCLUSION}

In summary, although imaging-based approaches to monitoring neurite outgrowth can provide detailed analyses of a number of features (e.g., number of extensions, branch points, and neurite lengths), these methods typically involve extensive time and labor to prepare samples, then image and analyze them. Here we report the development of a quick and simple indirect method for simultaneously measuring neurite outgrowth (via a cell membrane stain) and cell health (via a cell-permeable esterase substrate) in the same sample. This simple dual-color, dye-based staining method is not only suitable for microscopic visualization, but also enables rapid, unbiased microplate reader quantification. Here we described examples from testing this approach with monocultures (PC-12 derived Neuroscreen-1 cells) and two cultures (isolated rat cortex neurons and iPSC-derived iCell Neurons) that are highly enriched for neuronal cells. However, it is important to point out that since the dyes used in this approach are not neuron-specific this approach is not recommended for measuring neurite outgrowth in highly heterogeneous, mixed cultures of neuronal cells with other cell types. Instead, neurite outgrowth detection methods that stain for specific neuronal markers (e.g., beta-III tubulin or MAP2) should be used for mixed cultures, as well as for confirmation studies when applying nonspecific reporters such as the dyes described in this work. Nevertheless, because the assay approach described here (to report on neurite outgrowth and neural cell viability) is non-specific, one can envision that this same reporter dye combination could be useful for indirectly measuring additional cellular processes that affect changes in cell membrane content and/or cell viability.

\section{ABBREVIATIONS}

$\mathrm{EC}_{50}=50 \%$ effective concentration;
$\mathrm{IC}_{50}=50 \%$ inhibitory concentration;
$\mathrm{ICC}=$ immunocytochemistry;
$\mathrm{iPSC}=$ induced pluripotent stem cell;
$\mathrm{NGF}=$ nerve growth factor;
$\mathrm{RFU}=$ relative fluorescence unit

CONFLICT OF INTEREST

The authors are employees of Life Technologies, now part of Thermo Fisher Scientific.

\section{ACKNOWLEDGEMENTS}

We are grateful to Cellular Dynamics International for providing the iCell Neurons. We thank Jun Wang and Justin Wetter for excellent technical support. We also wish to thank Kun Bi, Shawn Honeyager, Robert Horton and Deborah Tieberg for their support, review and feedback relating to this work.

\section{REFERENCES}

[1] Harrill JA, Robinette BL, Freudenrich T, Mundy WR. Use of high content image analyses to detect chemical-mediated effects on neurite sub-populations in primary rat cortical neurons. Neurotoxicol 2013; 34: 61-73.

[2] Radio NM, Mundy WR. Developmental neurotoxicity testing in vitro: models for assessing chemical effects on neurite outgrowth. Neurotoxicol 2008; 29: 361-76.

[3] More SV, Koppula S, Kim IS, Kumar H, Kim BW, Choi DK. The role of bioactive compounds on the promotion of neurite outgrowth. Molecules 2012; 17: 6728-53.

[4] Xu X, Lei Y, Luo J, et al. Prevention of beta-amyloid induced toxicity in human iPS cell-derived neurons by inhibition of Cyclindependent kinases and associated cell cycle events. Stem Cell Res 2013; 10: 213-27.

[5] Harrill JA, Mundy WR. Quantitative assessment of neurite outgrowth in PC12 cells. Methods Mol Biol 2011; 758: 331-48.

[6] Radio NM, Breier JM, Shafer TJ, Mundy WR. Assessment of chemical effects on neurite outgrowth in PC12 cells using high content screening. Toxicol Sci 2008; 105: 106-18.

[7] Meijering E. Neuron tracing in perspective. Cytometry A 2010; 77 : 693-704.

[8] Yeyeodu ST, Witherspoon SM, Gilyazova N, Ibeanu GC. A rapid, inexpensive high throughput screen method for neurite outgrowth. Curr Chem Genom 2010; 4: 74-83.

[9] Ho SY, Chao CY, Huang HL, Chiu TW, Charoenkwan P, Hwang E. NeurphologyJ: an automatic neuronal morphology quantification method and its application in pharmacological discovery. BMC Bioinform 2011; 12: 230.

[10] Meijering E, Jacob M, Sarria JC, Steiner P, Hirling H, Unser M. Design and validation of a tool for neurite tracing and analysis in fluorescence microscopy images. Cytometry A 2004; 58: 167-76.

[11] Coder DM. Assessment of cell viability. Curr Protoc Cytom 2001; Chapter 9: Unit 9.2.

[12] Burroughs SL, Duncan RS, Rayudu P, et al. Plate reader-based assays for measuring cell viability, neuroprotection and calcium in primary neuronal cultures. J Neurosci Methods 2012; 203: 141-5. 
[13] Zhang JH, Chung TD, Oldenburg KR. A Simple Statistical Parameter for Use in Evaluation and Validation of High Throughput Screening Assays. J Biomol Screen 1999; 4: 67-73.

[14] Hancock MK, Lebakken CS, Wang J, Bi K. Multi-pathway cellular analysis of compound selectivity. Mol Biosyst 2010; 6: 1834-43.

[15] Haythornthwaite A, Stoelzle S, Hasler A, et al. Characterizing human ion channels in induced pluripotent stem cell-derived neurons. J Biomol Screen 2012; 17: 1264-72.
[16] Chase L, Strathman M, Grinager J, et al. Development and characterization of human iPSC-derived neurons for drug discovery applications. Biotechniques Poster Hall 2012. Available from URL: http://www.biotechniques.com/Posters/Cell_and_Tissue_Analysis/ Development-and-Characterization-of-Human-iPSC-derivedNeurons-for-Drug----Discovery-Applications/biotechniques329002.html

(C) Hancock et al.; Licensee Bentham Open.

This is an open access article licensed under the terms of the Creative Commons Attribution Non-Commercial License (http://creativecommons.org/licenses/by-nc/3.0/) which permits unrestricted, non-commercial use, distribution and reproduction in any medium, provided the work is properly cited. 